\title{
EDITORIAL
}

\section{It's microbiology, citizens}

\author{
The rise of the 'citizen science' movement in microbiology provides an opportunity for public \\ engagement and the chance to gather essential data on a large scale.
}

Those of you who follow the journal's Twitter feed (@NatureRevMicro) will have noticed we decamped recently to the annual jamboree that is the American Society for Microbiology (ASM) General Meeting in Denver, Colorado, USA, which this year attracted more than 5,000 scientists. Meetings on this scale are not for everyone - numerous parallel sessions in cavernous conference centres don't make for an intimate experience - but the meeting schedule has been revamped in recent years, and although of course there are still multiple sessions at any one time, there is much less redundancy than before, and the buzz of the exhibition hall and poster sessions, combined with the thrill of bumping into old friends and making new ones, is hard to beat.

One of the most talked-about sessions this year on Twitter and in personal encounters - was a session on 'citizen microbiology'. There is a long tradition in science of amateur practitioners making amazing discoveries (think Charles Darwin and Gregor Mendel, for example), but in the twentieth century this practice fell away. Over the past decade, however, powered by digital technology, the involvement of the public in largescale scientific projects has greatly increased with the emergence of the 'citizen science' movement. One of the longest running, e-Bird, which was started more than 10 years ago by the Cornell Lab of Ornithology (Ithaca, New York, USA) in collaboration with the National Audubon Society, allows birdwatchers to upload their observations to a central database, which now contains in excess of 100 million records and facilitates analysis of the abundance and distribution of birds worldwide. Over time, the database has evolved, and it now functions as a type of social network, allowing users to maintain their own lists and compare them to those of others. Other longrunning citizen science projects include SETI@home, one of the first distributed-computing projects, which uses internet-connected computers to analyse radio signals to search for extraterrestrial life and has more than 5 million registered participants.

The ASM session featured presentations on some of the citizen science-type projects that are currently operational in microbiology. It included projects that involve individuals who might be best referred to as 'novice' scientists, such as high-school students and university undergraduates. One of the best examples around is
Graham Hatfull's bacteriophage diversity and evolution research at the University of Pittsburgh Bacteriophage Institute (Pennsylvania, USA). The PHIRE (Phage Hunters Integrating Research and Education) programme (and an expanded programme that is part of the Howard Hughes Medical Institute Science Education Alliance initiative) turns undergraduate and high-school students into 'phage hunters'. The students isolate mycobacteriophages from soil and then annotate and analyse the resulting genome sequences. Involving early-stage students in such discovery-driven projects, rather than in more conventional laboratory projects that run year after year and have a defined outcome, captures their attention (particularly as students are allowed to name any novel phages) and provides invaluable insights into the scientific process and the nature of scientific inquiry. The students are also involved in reporting the results; more than 220 novel mycobacteriophages have been characterized in this way, and in a recent paper co-authored by the student consortia, a new model to account for the diversity of mycobacteriophages was proposed ${ }^{1}$.

Many newer citizen microbiology projects were also discussed. The Wildlife of our Homes project is headed by Rob Dunn at the University of North Carolina (Raleigh, USA). More than 1,000 participants in the USA registered for this project to characterize the microbial communities present in domestic homes, which involved taking swabs from nine specific household surfaces, including the toilet seat, kitchen chopping board and pillowcase. The results of the analysis of a small subset of samples were recently published ${ }^{2}$. Other interesting projects mentioned were the Belly Button Biodiversity project, the Earth Microbiome project and two gut microbiome projects, American Gut and $\mu$ biome.

Given the ease with which samples can be collected, it is perhaps surprising that there are not more citizen microbiology projects on the go, so one aim of the ASM session was to publicize the ongoing studies and inspire new ones, and hopefully that will have succeeded. Citizen microbiology projects are an ideal way to bring microbiology to a much wider audience - after all, who doesn't want to know what's lurking in their belly button?

\footnotetext{
. Jacobs-Sera, D. et al. On the nature of mycobacteriophage diversity and host preference. Virology 434, 187-201 (2012).

. Dunn, R. R. et al. Home life: factors structuring the bacterial diversity found within and between homes. PLOS ONE 8, e64133 (2013).
} 